\title{
HOLOMORPHIC FUNCTIONS ON NUCLEAR SPACES
}

\author{
BY
}

\section{PHILIP J. BOLAND}

ABSTRACT. The space $H(E)$ of holomorphic functions on a quasicomplete nuclear space is investigated. If $H(E)$ is endowed with the compact open topology, it is shown that $H(E)$ is nuclear if and only if $E^{\prime}$ (continuous dual of $E$ ) is nuclear. If $E$ is a $D F N$ (dual of a Fréchet nuclear space) and $F$ is a closed subspace of $E$, then the restriction mapping $H(E) \rightarrow H(F)$ is a surjective strict morphism.

1. Topologies on $H(E)$. In this article, $E$ will denote a quasi-complete nuclear locally convex space over the complex numbers $C$. $E^{\prime}$ will denote the continuous dual of $E$ endowed with the strong topology (topology of uniform convergence on bounded subsets of $E$ ). In a quasi-complete nuclear space $E$, bounded subsets are relatively compact. Therefore the strong topology on $E^{\prime}$ coincides with the Mackey topology (topology of uniform convergence on convex compact subsets of $E$ ). If $U$ is a convex balanced neighborhood of 0 in $E, p_{U}$ will denote its Minkowski functional.

If $K$ is a convex balanced compact subset of $E,[K]$ will denote the linear span of $K$ in $E$. If $x \in[K]$, then $p_{K}(x)=\inf \{\lambda: x \in \lambda K$ and $\lambda>0\}$. The polar $K^{\circ}$ of $K$ is the set $\left\{\varphi: \varphi \in E^{\prime}\right.$ where $\left.|\varphi|_{K}=\sup _{x \in K}|\varphi(x)| \leqslant 1\right\}$. As $K$ varies through the convex balanced compact subsets of $E$, the sets $K^{\circ}$ form a base of neighborhoods for 0 in $E^{\prime} . p_{K^{\circ}}$ is the seminorm defined on $E^{\prime}$ by

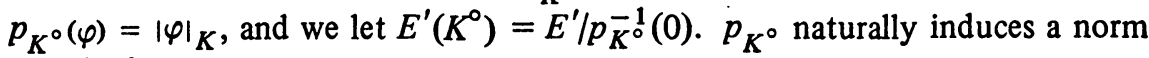
on $E^{\prime}\left(K^{\circ}\right)$ which will also be denoted $p_{K^{\circ}}$.

$E^{\prime}$ is a nuclear space if given any convex balanced compact subset $K$ of $E$, there exists another convex balanced compact subset $K_{1}$ of $E$ containing $K$ such that the mapping $E^{\prime}\left(K_{1}^{\circ}\right) \rightarrow E^{\prime}\left(K^{\circ}\right)$ is a nuclear mapping between normed spaces. $E^{\prime}\left(K_{1}^{\circ}\right) \rightarrow E^{\prime}\left(K^{\circ}\right)$ is nuclear if there exist $\left(a_{n}\right)_{n} \subseteq\left[K_{1}\right]$ and $\left(\varphi_{n}\right)_{n} \subseteq E^{\prime}$ such that for each $\varphi \in E^{\prime}, \varphi=\Sigma_{N}\left\langle a_{n}, \varphi\right\rangle \varphi_{n}$ (convergence in $E^{\prime}\left(K^{\circ}\right)$ ) and $\Sigma_{N} p_{K_{1}}\left(a_{n}\right) p_{K^{\circ}}\left(\varphi_{n}\right)=$ $M<+\infty$. By replacing $K_{1}$ by a sufficiently large multiple of itself, we may assume that $K \subseteq K_{1}$ and that $M<C$ where $C=1 / 4 \sup _{r \geqslant 1}\left(r^{r} / r !\right)^{1 / r}$.

Definition 1.1. $L\left({ }^{m} E\right), P\left({ }^{m} E\right)$ will denote, respectively, the continuous $m$-linear forms and the continuous $m$-homogeneous polynomials on $E$. For each 
compact set $K \subseteq E$ and $A \in L\left({ }^{m} E\right)$ (respectively $p \in P\left({ }^{m} E\right)$ ), let $\epsilon_{K}(A)=$ $\sup _{x_{i} \in K}\left|A\left(x_{1}, \ldots, x_{m}\right)\right|$ (respectively $\left.\epsilon_{K}(p)=\sup _{x \in K}|p(x)|\right)$. $\epsilon$ will be used to denote the topologies on $L\left({ }^{m} E\right)$ and $P\left({ }^{m} E\right)$ generated by all seminorms of the type $\epsilon_{K}$ as $K$ varies through the compact subsets of $E . L_{s}\left({ }^{m} E\right)$ will denote the continuous symmetric $m$-linear forms on $E$, and $P_{f}\left({ }^{m} E\right)$ will denote the continuous $m$-homogeneous polynomials of finite type on $E\left(p \in P\left({ }^{m} E\right)\right.$ is of finite type if $p$ can be represented in the form $p=\Sigma_{i=1}^{n} \varphi_{i}^{m}$ where $\left.\varphi_{i} \in E^{\prime}, i=1, \ldots, n\right)$.

Proposition 1.1. Let $E$ be quasi-complete and nuclear, and $E^{\prime}$ nuclear. Then $L\left({ }^{m} E\right), \epsilon$ is a nuclear space.

Proof. Let $K$ be a convex balanced compact subset of $E$. Since $E^{\prime}$ is nuclear there exists a convex balanced compact subset $K_{1}$ of $E$ containing $K$ such that the mapping $E^{\prime}\left(K_{1}^{\circ}\right) \rightarrow E^{\prime}\left(K^{\circ}\right)$ is nuclear with nuclear norm $<C$. Hence let $\left(a_{n}\right)_{n} \subseteq\left[K_{1}\right]$ and $\left(\varphi_{n}\right) \subseteq E^{\prime}$ be such that for each $\varphi \in E^{\prime}, \varphi=\Sigma_{N}\left\langle a_{n}, \varphi\right\rangle \varphi_{n}$ (convergence in $E^{\prime}\left(K^{\circ}\right)$ ) and $\Sigma_{N} p_{K_{1}}\left(a_{n}\right) p_{K^{\circ}}\left(\varphi_{n}\right)=M<C$. The claim is that the identity mapping $I_{K_{1}}^{m}, K: L\left({ }^{m} E\right), \epsilon_{K_{1}} \rightarrow L\left({ }^{m} E\right), \epsilon_{K}$ is nuclear and that its nuclear norm is $\leqslant M^{m}$.

Let $A \in L\left({ }^{m} E\right)$. From Boland [2], we know that $A$ may be represented in the form $A\left(x_{1}, \ldots, x_{m}\right)=\Sigma_{i=1}^{\infty}\left\langle x_{1}, \psi_{i 1}\right\rangle \cdots\left\langle x_{m}, \psi_{i m}\right\rangle$ where each $\psi_{i j}$ $\in E^{\prime}$ and for some convex balanced neighborhood $U$ of 0 in $E$ the series $\Sigma_{i=1}^{\infty} p_{U^{\circ}}\left(\psi_{i 1}\right) \cdots p_{U^{\circ}}\left(\psi_{i m}\right)$ converges.

Therefore

$$
\begin{aligned}
A & =\sum_{i=1}^{\infty}\left\langle\cdot, \psi_{i 1}\right\rangle \cdots\left\langle\cdot, \psi_{i m}\right\rangle \\
& =\sum_{i=1}^{\infty}\left\langle\cdot, \sum_{N}\left\langle a_{n}, \psi_{i 1}\right\rangle \varphi_{n}\right\rangle \cdots\left\langle\cdot, \sum_{N}\left\langle a_{n}, \psi_{i m}\right\rangle \varphi_{n}\right\rangle \\
& =\sum_{i=1}^{\infty} \sum_{n_{1}, \ldots, n_{m}}\left\langle a_{n_{1}}, \psi_{i 1}\right\rangle \cdots\left\langle a_{n_{m}}, \psi_{i m}\right\rangle \varphi_{n_{1}} \cdots \varphi_{n_{m}} \\
& =\sum_{n_{1}, \ldots, n_{m}}\left[\sum_{i=1}^{\infty}\left\langle a_{n_{1}}, \psi_{i 1}\right\rangle \cdots\left\langle a_{n_{m}}, \psi_{i m}\right\rangle\right] \varphi_{n_{1}} \cdots \varphi_{n_{m}} \\
& =\sum_{n_{1}, \ldots, n_{m}}\left\langle A, a_{n_{1} \ldots n_{m}}\right\rangle \varphi_{n_{1}} \cdots \varphi_{n_{m}}
\end{aligned}
$$

where $\left\langle A, a_{n_{1} \ldots n_{m}}\right\rangle=\Sigma_{i=1}^{\infty}\left\langle a_{n_{1}}, \psi_{i 1}\right\rangle \cdots\left\langle a_{n_{m}}, \psi_{i m}\right\rangle$.

Furthermore if $U_{1}$ is the unit ball in $L\left({ }^{m} E\right), \epsilon_{K_{1}}$ and $p_{U_{1}^{\circ}}\left(a_{n_{1} \ldots n_{m}}\right)=$ $\sup _{A \in U_{1}}\left|\left\langle A, a_{n_{1} \ldots n_{m}}\right\rangle\right|$, then 


$$
\begin{aligned}
\sum_{n_{1}, \ldots, n_{m}} & p_{U_{1}^{\circ}}\left(a_{n_{1} \ldots n_{m}}\right) \epsilon_{K}\left(\varphi_{n_{1}} \cdots \varphi_{n_{m}}\right) \\
& \leqslant \sum_{n_{1}, \ldots, n_{m}} p_{K_{1}}\left(a_{n_{1}}\right) \cdots p_{K_{1}}\left(a_{n_{m}}\right) \epsilon_{K}\left(\varphi_{n_{1}}\right) \cdots \epsilon_{K}\left(\varphi_{n_{m}}\right) \leqslant M^{m}
\end{aligned}
$$

This shows that $I_{K_{1}, K}^{m}$ is nuclear with nuclear norm

$$
\gamma\left(I_{K_{1}, K}^{m}\right) \leqslant M^{m}=\left(\sum_{N} p_{K_{1}}\left(a_{n}\right) p_{K^{\circ}}\left(\varphi_{n}\right)\right)^{m} .
$$

Corollary 1.1. $L_{s}\left({ }^{m} E\right), \epsilon$ is nuclear.

Corollary 1.2. $\left.P^{m} E\right), \epsilon$ is nuclear.

Proof. $P\left({ }^{m} E\right), \epsilon$ and $L_{s}\left({ }^{m} E\right), \epsilon$ are homeomorphic via the mapping $A$ $\in L_{s}\left({ }^{m} E\right) \rightarrow p_{A} \in P\left({ }^{m} E\right)$ where $p_{A}(x)=A(x, \ldots, x)$. Hence Corollary 1.2 follows from Corollary 1.1.

As in the proof of Proposition 1.1, it may be shown that the mapping $I_{K_{1}, K}^{m}: P\left({ }^{m} E\right), \epsilon_{K_{1}} \rightarrow P\left({ }^{m} E\right), \epsilon_{K}$ is nuclear and its nuclear norm $\gamma\left(I_{K_{1}, K}^{m}\right) \leqslant$ $m^{m} M^{m} / m$ !. This follows from elementary properties of compositions of nuclear and continuous mappings, and the following sequence of continuous mappings:

$$
\begin{aligned}
P\left({ }^{m} E\right), \epsilon_{K_{1}} & \rightarrow L_{s}\left({ }^{m} E\right), \epsilon_{K_{1}} \rightarrow L\left({ }^{m} E\right), \epsilon_{K_{1}} \stackrel{I_{K_{1}}^{m}, K}{\longrightarrow} L\left({ }^{m} E\right), \epsilon_{K} \\
& \rightarrow L_{s}\left({ }^{m} E\right), \epsilon_{K} \rightarrow P\left({ }^{m} E\right), \epsilon_{K} .
\end{aligned}
$$

(See Nachbin [5].)

DEFINITION 1.2. $H(E)$ will denote the space of all C-valued holomorphic functions on $E$. For each compact subset $K$ of $E, \epsilon_{K}$ will denote the seminorm defined on $H(E)$ by $\epsilon_{K}(f)=\sup _{x \in K}|f(x)|$. $\epsilon$ will denote the topology generated by all seminorms of the form $\epsilon_{K}$. $\epsilon$ is the compact open topology.

THEOREM 1.1. Let $E$ be quasi-complete and nuclear, and $E^{\prime}$ nuclear. Then $H(E), \epsilon$ is nuclear.

Proof. Let $K$ be a given compact set, and choose $K_{1}$ as in Corollary 1.2. We know that for each $m \geqslant 1$,

$$
P\left({ }^{m} E\right), \epsilon_{K_{1}} \stackrel{I_{K_{1}}^{m}, K}{\longrightarrow} P\left({ }^{m} E\right), \epsilon_{K}
$$

is nuclear with nuclear norm

$$
\gamma\left(I_{K_{1}}^{m}, K\right) \leqslant \frac{m^{m}}{m !} M^{m} \leqslant \frac{m^{m}}{m !}\left(1 / 4 \sup _{r \geqslant 1}\left(\frac{r^{r}}{r !}\right)^{1 / r}\right)^{m}<\frac{1}{2^{m}} .
$$

Hence for each $m \exists\left(a_{m n}\right)_{n} \subseteq\left(P\left({ }^{m} E\right), \epsilon_{K_{1}}\right)^{\prime}$ and $\left(y_{m n}\right)_{n} \subseteq P\left({ }^{m} E\right), \epsilon_{K}$ such that for $p \in P\left({ }^{m} E\right), \epsilon_{K_{1}}$, 


$$
p=\sum_{n}\left\langle p, a_{m n}\right\rangle y_{m n} \quad \text { (convergence in } P\left({ }^{m} E\right), \epsilon_{K} \text { ) }
$$

and $\Sigma_{n} p_{U_{1}^{\circ}}\left(a_{m n}\right) \epsilon_{K}\left(y_{m n}\right)<1 / 2^{m}$ (where $U_{1}$ is the unit ball in $P\left({ }^{m} E\right), \epsilon_{K_{1}}$ ). Now each $a_{m n}$ may be extended to $\widetilde{a}_{m n}$ which is continuous on $H(E), \epsilon$, by defining

$$
\tilde{a}_{m n}(f)=a_{m n}\left(\hat{d}^{m} f(0) / m !\right) .
$$

Hence, given $f \in H(E), \epsilon_{K_{1}}$,

$$
\begin{aligned}
f & =\sum_{m=0}^{\infty} \frac{\hat{d}^{m} f(0)}{m !} \\
& =\sum_{m=0}^{\infty} \sum_{n=0}^{\infty}\left\langle\frac{\hat{d}^{m} f(0)}{m !}, a_{m n}\right\rangle y_{m n} \\
& =\sum_{m=0}^{\infty} \sum_{n=0}^{\infty}\left\langle f, \tilde{a}_{m n}\right\rangle y_{m n}=\sum_{m, n \geqslant 0}\left\langle f, \tilde{a}_{m n}\right\rangle y_{m n} .
\end{aligned}
$$

Moreover, if $U_{1}$ is the unit ball in $H(E), \epsilon_{K_{1}}$, then

$$
\sum_{m, n \geqslant 0} p_{U_{1}^{\circ}}\left(\tilde{a}_{m n}\right) \epsilon_{K}\left(y_{m n}\right) \leqslant \sum_{m=0}^{\infty} \frac{1}{2^{m}}=2 .
$$

Therefore $H(E), \epsilon_{K_{1}} \rightarrow H(E), \epsilon_{K}$ is nuclear.

COROllary 1.3. Let $E$ be a nuclear quasi-complete space. Then $H(E)$, $\epsilon$ is nuclear $\Leftrightarrow E^{\prime}$ is nuclear.

COROLLARY 1.4. Let E be a DFN space (the strong dual of a Fréchet nuclear space). Then $H(E), \epsilon$ is a Fréchet nuclear space. In particular it is reflexive and separable.

ExAmples. $H(D), H\left(D^{\prime}\right), H(E), H(S), H\left(\Sigma_{N} C\right)$ are all nuclear spaces when endowed with the compact open topology. $H\left(E^{\prime}\right), H\left(S^{\prime}\right), H\left(H^{\prime}(C)\right)$ are Fréchet nuclear spaces when endowed with the compact open topology.

Note. In Barroso, Matos and Nachbin [1] it has been shown that when $E$ is a Silva space, all of the standard topologies $\tau_{\delta}, \tau_{\omega}, \tau_{0}(=\epsilon)$ coincide on $H(E)$. Hence in particular we know that if $E$ is a DFN space, then all of the standard topologies $\tau_{\delta}, \tau_{\omega}, \tau_{0}$ on $H(E)$ are equivalent Fréchet nuclear topologies.

2. Polynomials on $E$.

Proposition 2.1. Suppose $E$ is a DFN space. Then given any $p \in P\left({ }^{m} E\right)$, 
$p$ may be represented in the form $p=\Sigma_{i=1}^{\infty} \varphi_{i}^{m}\left(\varphi_{i} \in E^{\prime}\right)$ where for each compact subset $K$ of $E, \Sigma_{i=1}^{\infty}\left|\varphi_{i}^{m}\right|_{K}<+\infty$.

PRoof. Since $E$ is a DFN space, there exists an increasing sequence $\left(K_{n}\right)_{n}$ of convex compact subsets of $E$ such that given any compact $K$, then for some $n, K \subseteq K_{n}$. Now we know that $P_{f}\left({ }^{m} E\right)$ is dense in $P\left({ }^{m} E\right)$ for the compact open topology. In Boland [2] it was shown that the compact open topology ( $\epsilon$ topology) and the $\pi$ topology coincide on $P\left({ }^{m} E\right) . \pi$ is the topology generated by all $\pi_{K}$, where $\pi_{K}$ is defined on $P_{f}\left({ }^{m} E\right)$ in the following manner:

$$
\pi_{K}(q)=\inf \left\{\sum_{i=1}^{s}\left|\varphi_{i}^{m}\right|_{K}: q=\sum_{i=1}^{s} \varphi_{i}^{m}\right\} \text {. }
$$

Now suppose $p \in P\left({ }^{m} E\right)$. Then by these remarks there exists a sequence $\left(p_{r}\right)_{r} \subseteq$ $\boldsymbol{P}_{f}\left({ }^{m} E\right)$ which converges to $p$ (in both the $\pi$ and $\epsilon$ topologies). In fact (by appropriately choosing a subsequence) we may assume that for $r \geqslant n$, $\pi_{K_{n}}\left(p_{r}-p_{r-1}\right)<1 / 2^{r}$ for each $n$. Let $q_{1}=p_{1}$ and $q_{r}=p_{r}-p_{r-1}$ for $r>1$. Then $\Sigma_{s=1}^{r} q_{s}=p_{r} \rightarrow p$ as $r \rightarrow \infty$. For each $r>1$ choose $\varphi_{r 1}, \ldots, \varphi_{r j_{r}}$ such that $q_{r}=\Sigma_{i=1}^{j_{r}} \varphi_{r i}^{m}$ and $\Sigma_{i=1}^{j_{r}}\left|\varphi_{r i}^{m}\right|_{K_{r}}<1 / 2^{r}$. Now for each $x \in E, \Sigma_{r=1}^{\infty} \Sigma_{i=1}^{j_{r}} \varphi_{r i}^{m}(x)=$ $p(x)$, and furthermore given a compact subset $K$ of $E, K \subseteq K_{s}$ for some $s$ and hence

$$
\begin{aligned}
\sum_{r=1}^{\infty} \sum_{i=1}^{j_{r}}\left|\varphi_{r i}^{m}\right|_{K} & \leqslant \sum_{r=1}^{\infty} \sum_{i=1}^{j_{r}}\left|\varphi_{r i}^{m}\right|_{K_{s}} \\
& \leqslant \sum_{r=1}^{s} \sum_{i=1}^{j_{r}}\left|\varphi_{r i}^{m}\right|_{K_{s}}+\sum_{r=s+1}^{\infty} \sum_{i=1}^{j_{r}}\left|\varphi_{r i}^{m}\right|_{K_{r}} \\
& \leqslant \sum_{r=1}^{s} \sum_{i=1}^{j_{r}}\left|\varphi_{r i}^{m}\right|_{K_{s}}+\sum_{r=s+1}^{\infty} \frac{1}{2^{r}}<\infty
\end{aligned}
$$

Hence $p=\Sigma_{r=1}^{\infty} \Sigma_{i=1}^{j_{r}} \varphi_{r i}^{m}$ is a representation of the desired form.

3. Extending holomorphic functions. In this section $E$ will be assumed to be a DFN space.

LEMMA 3.1. For each compact $K \subseteq E, \tilde{\pi}_{K}(f)=\Sigma_{n=0}^{\infty} \pi_{K}\left(\hat{d}^{n} f(0) / n !\right)$ defines a seminorm on $H(E)$. Furthermore the $\pi$ topology on $H(E)$ generated by all such $\tilde{\pi}_{K}$ is equivalent to the $\epsilon$ (compact open) topology on $H(E)$.

Proof. $H(E), \epsilon$ is a Fréchet nuclear space. Hence by Dineen [3, Proposition 1.3], the topology $\epsilon$ on $H(E)$ is that generated by all seminorms $\widetilde{\epsilon}_{K}$ where $\widetilde{\epsilon}_{K}(f)=\Sigma_{n=0}^{\infty} \epsilon_{K}\left(\hat{d}^{n} f(0) / n !\right)$. By Boland [2, Corollary 1.5], given a compact 
$K \subseteq E$, there exists a compact $K_{1}$ (such that for some $\alpha>0, K \subseteq \alpha K_{1}$ ) and a constant $C_{K}>0$ such that $\pi_{K}(p) \leqslant C_{K}^{m} \epsilon_{K_{1}}(p)$ for each $p \in P\left({ }^{m} E\right), m=0,1$, ... By appropriately choosing $K_{1}$, we may assume $C_{K}=1$. Hence

$$
\tilde{\pi}_{K}(f)=\sum_{n=0}^{\infty} \pi_{K}\left(\frac{\hat{d}^{n} f(0)}{n !}\right) \leqslant \sum_{n=0}^{\infty} \epsilon_{K_{1}}\left(\frac{\hat{d}^{n} f(0)}{n !}\right)=\tilde{\epsilon}_{K_{1}}(f) .
$$

Hence $\tilde{\pi}_{K}$ is a seminorm on $H(E)$, and $\pi$ is at least as fine as $\epsilon$. Since $\tilde{\epsilon}_{K}(f) \leqslant$ $\tilde{\pi}_{K}(f)$, it follows that $\epsilon$ and $\pi$ coincide on $H(E)$.

LEMMA 3.2. Let $F$ be a closed subspace of the DFN space $E$. Then the restriction mapping $R: H(E) \rightarrow H(F)$ is almost open (given any neighborhood $U$ of 0 in $H(E)$, there exists a neighborhood $V$ of 0 in $H(F)$ such that $V \subseteq \overline{R(U)})$.

Proof. Since $F$ is closed, $F$ is also a $D F N$ space. Hence $P\left({ }^{m} E\right), P\left({ }^{m} F\right)$ $(m=0,1,2, \ldots)$, and $H(E), H(F)$ are Fréchet nuclear spaces.

Let $U=\left\{\widetilde{f}: \widetilde{f} \in H(E): \widetilde{\pi}_{K}(\widetilde{f})<\alpha\right\}$ where $\alpha<1$. We will show that $\overline{R(U)}$ is a neighborhood of 0 in $H(F)$.

The restriction mapping $R: E^{\prime} \rightarrow F^{\prime}$ is open, continuous and onto. Hence there exists a compact $K^{\prime}$ in $F$ such that $\left\{\varphi: \varphi \in F^{\prime},|\varphi|_{K^{\prime}}<1\right\} \subseteq R\left\{\widetilde{\varphi}: \widetilde{\varphi} \in E^{\prime}\right.$, $\left.|\widetilde{\varphi}|_{K}<\alpha\right\}$. In particular given $\varphi \in F^{\prime}$ and $\beta>0$ such that $|\varphi|_{K^{\prime}}<\beta$, there exists an extension $\tilde{\varphi} \in E^{\prime}$ of $\varphi$ such that $|\widetilde{\varphi}|_{K}<\alpha \beta$.

Let $m$ be any integer $\geqslant 2$. Suppose $p \in P_{f}\left({ }^{m} F\right)$ such that $\pi_{K^{\prime}}(p)<\beta$. Then $p$ may be written in the form $p=\Sigma_{i=1}^{r} \varphi_{i}^{m}$ where $\varphi_{i} \in F^{\prime} \forall i$, and $\Sigma_{i=1}^{r}\left|\varphi_{i}^{m}\right|_{K^{\prime}}<\beta$. For each $i$, let $\widetilde{\varphi}_{i}$ be an extension of $\varphi_{i}$ to $E$ such that $\widetilde{\varphi}_{i} \in$ $E^{\prime}$ and $\Sigma_{i=1}^{r}\left|\widetilde{\varphi}_{i}^{m}\right|_{K}<\alpha^{m} \beta$. Hence by letting $\widetilde{p}=\Sigma_{i=1}^{r} \widetilde{\varphi}_{i}^{m}$ we have $\widetilde{p} \in P_{f}\left({ }^{m} E\right)$ and $\pi_{K}(\widetilde{p})<\alpha^{m} \beta$. Therefore if $w^{m}=\left\{\widetilde{p}: \widetilde{p} \in P\left({ }^{m} E\right), \pi_{K}(\widetilde{p})<\alpha^{m}\right\}$, then $\left\{p: p \in P_{f}\left({ }^{m} F\right), \pi_{K^{\prime}}(p)<1\right\} \subseteq R\left(w^{m}\right)$. Hence $\left\{p: p \in P\left({ }^{m} F\right), \pi_{K^{\prime}}(p)<1\right\} \subseteq$

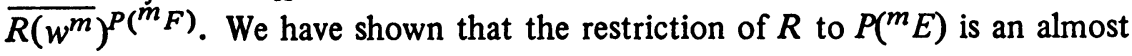
open mapping onto a dense subset of $P\left({ }^{m} F\right)$. Hence it is an open mapping onto $P\left({ }^{m} F\right)$ (Horvath [4, Theorem 3.17.2, Proposition 3.17.12]) and we may assume

$$
\left\{p: p \in P\left({ }^{m} F\right), \pi_{K^{\prime}}(p)<1\right\} \subseteq R\left\{\widetilde{p}: \widetilde{p} \in P\left({ }^{m} E\right), \pi_{K}(\widetilde{p})<\alpha^{m}\right\} .
$$

In particular given $p \in P\left({ }^{m} F\right)$ and $\beta>0$ such that $\pi_{K^{\prime}}(p)<\beta$, there exists an extension $\widetilde{p} \in P\left({ }^{m} E\right)$ of $p$ such that $\pi_{K}(\widetilde{p})<\alpha^{m} \beta$. Now let

$$
V=\left\{f: f \in H(F), \sum_{m=0}^{\infty} \pi_{K^{\prime}}\left(\frac{\hat{d}^{m} f(0)}{m !}\right)<\beta=\frac{\alpha(1-\alpha)}{2}\right\} .
$$

$V$ is a neighborhood of 0 in $H(F)$, and we will show that $V \subseteq \overline{R(U)}$. Suppose $f \in V$. Since $\Sigma_{m=0}^{r} \hat{d}^{m} f(0) / m ! \rightarrow f$ as $r \rightarrow \infty$, to show that $f \in \overline{R(U)}$ it suffices to show that $\Sigma_{n=0}^{r} \hat{d}^{n} f(0) / n ! \in R(U)$ for each $r$. For each $m=1,2, \ldots$, let $\widetilde{p}_{m} \in P\left({ }^{m} E\right)$ be an extension of $\hat{d}^{m} f(0) / m$ ! such that $\pi_{K}\left(\widetilde{p}_{m}\right) \leqslant \alpha^{m} \beta$. Then 


$$
\sum_{m=0}^{r} \pi_{K}\left(\widetilde{p}_{m}\right) \leqslant \sum_{m=0}^{r} \alpha^{m} \beta<\beta /(1-\alpha)<\alpha .
$$

Hence $\Sigma_{m=0}^{r} \widetilde{p}_{m} \in U$ and $\Sigma_{m=0}^{r} \hat{d}^{m} f(0) / m ! \in R(U)$.

THEOREM 3.1. Let $F$ be a closed subspace of the DFN space E. Then if $H(E), H(F)$ are endowed with the compact open topologies, the restriction mapping $R: H(E) \rightarrow H(F)$ is a strict morphism onto (i.e. continuous, open, linear and onto).

PROOF. $R$ is clearly a continuous linear mapping, and by Lemma 3.2 it is an almost open mapping with dense image. Since $H(E), H(F)$ are Fréchet spaces, it follows that $R$ is open and onto (see Horvath [4, Theorem 3.17.2, Proposition 3.17.12]).

COROLlary 3.1. Let $F$ be a closed subspace of the DFN space E. Then given $f \in H(F)$, there exists an extension $\tilde{f} \in H(E)$ of $f$.

\section{BIBLIOGRAPHY}

1. J. A. Barroso, M. C. Matos and L. Nachbin, On bounded sets of holomorphic mappings, Proc. 1973 Internat. Conf. on Infinite Dimensional Holomorphy, Lecture Notes in Math., vol. 364, Springer-Verlag, Berlin and New York, 1974.

2. P. J. Boland, Malgrange theorem for entire functions on nuclear spaces, Proc. 1973 Internat. Conf. on Infinite Dimensional Holomorphy, Lecture Notes in Math., vol. 364, Springer-Verlag, Berlin and New York, 1974.

3. S. H. Dineen, Holomorphic functions on locally convex topological vector spaces: I. Locally convex topologies on $H(U)$, Ann. Inst. Fourier Univ. (Grenoble) 23 (1973).

4. J. M. Horváth, Topological vector spaces and distributions. Vol. I, Addison-Wesley, Reading, Mass., 1966. MR 34 \#4863.

5. Leopoldo Nachbin, Topology on spaces of holomorphic mappings, Ergebnisse der Mathematik und ihrer Grenzgebiete, Band 47, Springer-Verlag, New York, 1969. MR 40 \#7787.

6. A. Pietsch, Nuclear locally convex spaces, Ergebnisse der Mathematik und ihrer Grenzgebiete, Band 66, Springer-Verlag, New York, 1972. IRELAND

DEPARTMENT OF MATHEMATICS, UNIVERSITY COLLEGE OF DUBLIN, DUBLIN,

Current address: Department of Mathematics, Le Moyne College, Le Moyne Heights, Syracuse, New York 13214 